\title{
Development of a questionnaire to understand the information needs of adolescent and young adult cancer survivors for life and long-term health following cancer treatment.
}

\author{
Devin Gantzios-Cros ${ }^{1}$, Tammy Sajdyk ${ }^{2}$, Jamie Renbarger ${ }^{2}$ \\ ${ }^{1}$ Indiana University School of Medicine; ${ }^{2}$ Indiana University School of Medicine, Department of \\ Pediatrics
}

Disease outcomes for adolescents and young adults (AYA: ages 15 - 39 years old) with cancer have improved significantly over the past four decades to over $80 \%$. AYA survivors are a unique population who have decades of life years following cancer treatment and are at very high risk for developing chronic medical and mental health conditions. Unfortunately, there is gap by providers in providing information regarding expectations of long-term medical, cognitive, and psychosocial issues that may arise during their lifetime. The goal of this project was to understand the educational/informational needs of this unique population. In order to understand the type of information AYAs would like to know post-treatment, such as exercise, nutrition, and more, we developed a survey addressing key topics. The process of collecting data has begun and will be collected for an additional five months. Subsequently, data will be analyzed by our team to determine key areas of interest. We will then utilize social media platforms to target AYA survivors and disseminate educational information addressing those areas of interest. The overarching hypothesize is that minimizing the health information gap and increasing knowledge about survivorship in the AYA cancer population via an accepted social media platform will lead to a more informed population of survivors that can better address their life long healthcare. 